\title{
LOS PROGRAMAS DE FORMACIÓN DE PADRES: UNA EXPERIENCIA EDUCATIVA
}

\author{
Isabel Bartau, Juana Maganto, Juan Etxeberría \\ Dpto. de Métodos de Investigación y Diagnóstico en Educación \\ Universidad del País Vasco
}

\section{La Formación de Padres}

En general, ha habido poco consenso sobre la definición de formación de padres (Hicks y Williams, 1981). Debido a ello, comprende diversidad de objetivos, contenidos y métodos (Brock, Oertwein y Coufal, 1993).

El término formación de padres indica un intento de acción formal con el objeto de incrementar la conciencia de los padres y la utilización de sus aptitudes y competencias parentales (Lamb y Lamb, 1978). La formación de los padres "forma parte de la educación de los niños y es un método para promover su desarrollo" (Cataldo, 1991:17) a través del incremento de la información, del conocimiento y las habilidades de los padres para atender las necesidades de los niños a lo largo de su desarrollo. La formación parental constituye una "acción educativa de sensibilización, de aprendizaje, de adiestramiento o de clarificación de los valores, las actitudes y las prácticas de los padres en la educación" (Boutin y Durning, 1997:24) que comprende "un proceso de desarrollo individual tendiente a perfeccionar las capacidades de sentir, de imaginar, de comprender, de aprender, de utilizar unos conocimientos" (Durning, (1995:39). O también se ha caracterizado como "conjunto de actividades voluntarias de aprendizaje por parte de los padres que tiene como objetivo proveer modelos adecuados de prácticas educativas en el contexto familiar y/o modificar y mejorar prácticas existentes con el objeto de promover comportamientos en los hijos y las hijas que son juzgados positivamente y erradicar los que se consideran negativos (Vila, 1997a). Por lo tanto el término formación de padres alude al desarrollo de habilidades de los padres y madres para educar a sus hijos.

Por otra parte, también se debate si estos programas tienen un carácter preventivo y/o remedial. Otras características que definen la formación de padres incluyen que su carácter es eminentemente preventivo y su objetivo es la mejora de las prácticas educativas en las familias de una población determinada a diferencia de otras formas de intervención individual con las familias (Pourtois, 1984). No obstante, si bien la educación acerca de la paternidad tiene una finalidad principalmente preventiva tiene un carácter tanto preventivo como remedial, y en los últimos 10 años, los padres, los tribunales de justicia, las iglesias y los centros de salud mental comunitarios consideran la educación acerca de la paternidad como el remedio para algunos de los problemas que más afectan a las familias (Brock, Oertwein y Coufal, 1993). Este carácter preventivo/remedial no sólo se constata en el contexto americano, sino que, en la actualidad, en nuestro contexto sociocultural del País Vasco (España), cada vez se demanda-oferta-orienta-prescribe-obliga la formación de padres desde ámbitos comunitarios como los Centros de Salud o los Tribunales de Justicia que orientan a los padres en proceso de divorcio hacia las clases de formación de padres para enseñarles a sus hijos a afrontar el divorcio o, en su caso, dictan en las sentencias de los padres abusivos que asistan a clases de formación de padres. 


\section{Justificación y evolución histórica}

En la actualidad, se recogen diversas razones o indicadores de la necesidad de educación para la paternidad que justifican los programas en este campo y que se presentan a continuación:

1. El deseo de los padres de desempeñar adecuadamente este rol, especialmente, en épocas de rápidos cambios en las que tienen que enfrentarse a circunstancias cambiantes, requiere revisar sus funciones parentales básicas y asumir nuevos roles (Brock, Oertwein y Coufal, 1993).

2. Los procesos de nuclerización familiar, la erosión de la comunidad, la diferenciación y especialización de roles, la distancia geográfica de las generaciones familiares, y el incremento del trabajo fuera del hogar (Harmin y Brim, 1980:14).

3. El incremento de la incidencia del divorcio y las familias reconstituídas, el abuso y negligencia infantil, los problemas emocionales y la alta incidencia del embarazo en la adolescencia (Hicks y Williams, 1981).

4. La creencia de los padres de que el ser padre en la actualidad es más duro que en el pasado, la preocupación por el consumo de drogas por parte de niños y adolescentes, los índices de suicidio en la adolescencia (Fine y Henry, 1991).

5. La entrada en la paternidad/maternidad conlleva amplias transformaciones en la vida de las personas dado que constituye un continuo y complejo proceso de adaptación al rol paterno (Cataldo, 1991) especialmente en las sucesivas fases de la paternidad que reflejan las nuevas responsabilidades que habrá que afrontar (Galinsky, 1981, cit. Wapner, 1993).

6. La educación de los niños y la educación de los padres son los instrumentos más asequibles y eficaces para incrementar la capacidad de las naciones para cubrir las necesidades / lograr las aspiraciones de desarrollo humano (Hobbs, Dokecki, Hoover-Dempsey, Moroney, Shayne y Weeks,1984).

Tavoillot (1982) recoge la historia de la Escuela de Padres fundada en París por Vérine en 1929 cuyas actuaciones se divulgaron con su Revista. Partiendo en sus comienzos de un carácter "moralizante" y "confesional", en 1942 adquiere un carácter más científico proponiéndose como objetivo "hacer comprender a los padres y futuros padres la gravedad de su papel, atrayendo la atención de éstos sobre los problemas que plantea la educación familiar" y creando un título de educador familiar (Tavoillot, 1982:264). La Escuela de Padres de Vérine tuvo gran influencia en el desarrollo de las escuelas de padres en España (Otero,1990). (...) "la necesidad de aportar ayuda a los padres para situarlos en mejores condiciones de asumir la función educativa" se deriva de que "la escuela en particular no deja de poner en evidencia los fracasos de la educación familiar" (Tavoillot, 1982:263). Tavoillot (1982:264-275) analiza la evolución de la metodología de la educación de padres desde 1949 hasta mediados de los años 70 como se puede apreciar en el cuadro nํㅜ 1 . 
Cuadro $\mathrm{n}^{\circ}$ 1: Evolución de los objetivos, métodos y rol del orientador en los programas de formación de padres (Basado en Tavoillot, 1982)

\begin{tabular}{|c|c|c|c|c|}
\hline ETAPA & OBJETIVO & METODO & $\begin{array}{l}\text { ROL DEL } \\
\text { EDUCADOR }\end{array}$ & $\begin{array}{l}\text { RELACION } \\
\text { EDUCADOR- } \\
\text { PADRES }\end{array}$ \\
\hline $\begin{array}{l}\text { NIÑOS- } \\
\text { PROBLEMA }\end{array}$ & $\begin{array}{l}\text { Suministrar } \\
\text { información sobre } \\
\text { los problemas de } \\
\text { los niños con el } \\
\text { fin de que los } \\
\text { padres, tras su } \\
\text { reflexión } \\
\text { razonamiento, y } \\
\text { cambien su } \\
\text { comportamiento }\end{array}$ & $\begin{array}{l}\text { Charlas, } \\
\text { Conferencias }\end{array}$ & $\begin{array}{l}\text { Especialista- } \\
\text { experto } \\
\text { Da consejos, } \\
\text { prescriber cómo } \\
\text { solucionar los } \\
\text { problemas de los } \\
\text { hijos. }\end{array}$ & Asimétrica \\
\hline $\begin{array}{l}\text { PADRES- } \\
\text { PROBLEMA }\end{array}$ & $\begin{array}{l}\text { Contribuir a que } \\
\text { los padres } \\
\text { descubran que el } \\
\text { origen de los } \\
\text { problemas de sus } \\
\text { hijos pueden } \\
\text { encontrarse en su } \\
\text { propio } \\
\text { comportamiento, } \\
\text { manifestación de } \\
\text { dificultades } \\
\text { personales o } \\
\text { conyugales de los } \\
\text { padres }\end{array}$ & $\begin{array}{l}\text { Entrevista } \\
\text { personal } \\
\text { Reunión en } \\
\text { pequeños grupos }\end{array}$ & $\begin{array}{l}\text { Especialista- } \\
\text { experto } \\
\text { Da consejos, } \\
\text { prescriber cómo } \\
\text { solucionar los } \\
\text { problemas de los } \\
\text { padres }\end{array}$ & Asimétrica \\
\hline $\begin{array}{l}\text { EDUCADOR- } \\
\text { PROBLEMA }\end{array}$ & $\begin{array}{lrr}\text { Contribuir } & \text { a } \\
\text { mejorar el } & \text { rol } \\
\text { parental } & \text { y } \\
\text { favorecer } & \text { la } \\
\text { autoconfianza } & y \\
\text { autocompetencia } \\
\text { parental }\end{array}$ & $\begin{array}{l}\text { Entrevista } \\
\text { personal } \\
\text { Reunión en } \\
\text { pequeños grupos }\end{array}$ & $\begin{array}{l}\text { Educador no } \\
\text { directivo } \\
\text { Estimula y confía } \\
\text { en la capacidad } \\
\text { de los padres } \\
\text { para ejercer su } \\
\text { función }\end{array}$ & Simétrica \\
\hline $\begin{array}{l}\text { SOCIEDAD } \\
\text { PROBLEMA }\end{array}$ & $\begin{array}{l}\text { Coordinar las } \\
\text { acciones } \\
\text { educativas y las } \\
\text { relaciones } \\
\text { familia-escuela- } \\
\text { comunidad }\end{array}$ & $\begin{array}{lr}\text { Integran } & \text { los } \\
\text { programas } & \text { de } \\
\text { formación } & \text { de } \\
\text { padres } & \text { en } \\
\text { programas } & \\
\text { escuela- } & \\
\text { comunidad } & \text { más } \\
\text { amplios } & \\
\end{array}$ & $\begin{array}{l}\text { Agente de } \\
\text { cambio, } \\
\text { facilitador en la } \\
\text { mediación } \\
\text { familia-escuela- } \\
\text { comunidad }\end{array}$ & Intersistémica \\
\hline
\end{tabular}

La evolución que presenta Tavoillot (1982), con gran acierto, a nuestro entender, se adelantó pronosticando la evolución de la pedagogía familiar hacia la época de la sociedad-problema. "Las condiciones de vida, los ritmos de trabajo impuestos a los padres, la insuficiencia de las instituciones 
que pudieran ayudarlos, o al menos reemplazarlos en cierto grado y en determinados momentos, ¿no origina todo esto dificultades imposibles de resolver por parte de la pedagogía familiar, por ilustrada que pueda estar por la psicología y el psicoanálisis? En otras palabras ¿puede la pedagogía familiar seguir centrándose en la familia, y continuar descartando el componente político en su más amplio sentido? La cuestión, desde luego, no nos incumbe a nosotros resolverla, pero tampoco podemos rehusar plantearla" (Tavoillot, 1982:275).

En la actualidad, en la etapa que Tavoillot denominó de la sociedad-problema, las relaciones entre paternidad/maternidad y la educación de los hijos, no es algo que dependa exclusivamente de los padres sino que, fundamentalmente por la influencia de la perspectiva ecosistémica, forman parte de un sistema de relaciones internas y externas con su entorno. El objetivo, por lo tanto, es coordinar las acciones educativas entre las familias, la escuela y la comunidad para contribuir conjuntamente al desarrollo y la educación de los niños/jóvenes. El rol de orientador, es el de agente de cambio y facilitador de las relaciones entre los agentes educativos de las diversas instancias. Los métodos, eminentemente preventivos pero atendiendo las dificultades y la diversidad, integran los programas de formación de padres en programas comunitarios más amplios para atender a las necesidades de los niños/jóvenes, familias, escuelas y comunidad considerando las circunstancias y recursos con que cuentan para el desarrollo de los programas.

En la actualidad, se diferencian tres modelos de formación en las Escuelas de Padres, el Modelo Informativo, el Modelo Instructivo y elModelo Social (Velázquez y Loscertales, 1987).

\section{Organización de los programas de formación de padres}

La organización de los programas de formación de padres y, en consecuencia, la delimitación de los objetivos, las áreas de contenido, los métodos y los procedimientos de evaluación varían dependiendo de las necesidades de los participantes, las características del contexto desde el cual parte la intervención, los enfoques teóricos que subyacen a los programas y los modelos de intervención adoptados por el orientador. A continuación, revisamos las características principales del desarrollo de estos programas.

\section{Objetivos}

La mayoría de los programas persiguen dos objetivos generales, estimular el desarrollo del niño y apoyar a los padres (información, desarrollo de habilidades, confianza..), objetivos que se concretan en los siguientes (Cataldo, 1991:117-131):

1. Informar, asesorar y orientar a los padres sobre el desarrollo, el aprendizaje y la socialización del niño.

2. Estimular su participación en el aprendizaje y experiencias escolares del niño.

3. Enseñarles técnicas y aptitudes específicas sobre el aprendizaje infantil y el control del comportamiento.

4. Prevenir problemas en el desarrollo del niño o en las relaciones familiares.

5. Ofrecer asesoramiento y rehabilitación a las familias que presenten problemas en el desarrollo de sus hijos o en las relaciones familiares. 
6. Asesorar a los padres de niños de educación especial.

7. Proporcionar apoyos sociales en la comunidad.

8. Estimular y apoyar las iniciativas de interés especial promovidas por los padres.

A pesar de la variedad de los objetivos de los programas de formación de padres, una revisión revela que se centran en una o varias de las siguientes áreas (Fine y Brownstein, 1983):

\section{A) Compartir información}

El orientador persigue estimular el compartir la información presentando hechos, conceptos e información teórica acerca de las diversas áreas y procesos educativo-interaccionales en la familia. Hay muchos libros, panfletos y cassettes sobre aspectos de la paternidad y el desarrollo de los hijos que permiten distribuir la información de forma sencilla y entretenida.

\section{B) Adquisición y desarrollo de habilidades}

El compartir información a menudo es insuficiente para conseguir cambios conductuales porque sólo decir a la gente lo que tiene que hacer nunca ha demostrado ser un vehículo educativo efectivo. Por ello, a la información suele incorporarse la construcción de habilidades. Suelen utilizarse las técnicas de role-playing, el modelado y el ensayo conductual para enseñar a los padres habilidades específicas y apoyar la información compartida. Se enseña a los padres cómo utilizar los "mensajes yo", la atención reflexiva, el modelado, el reforzarmiento, la estimulación, cómo realizar reuniones familiares, la negociación, el poner límites, la utilización del tiempo fuera, y "controlar" la conducta de sus hijos. Estos objetivos de adquisición de habilidades tienen la característica común de que pueden ser expresados en términos de conductas observables.

\section{C) Cambio de creencias}

Este tipo de objetivos persiguen, por un lado, desarrollar la autoconciencia y el autoconocimiento de los padres con respecto al propio estilo de paternidad, su génesis y su influencia en el desarrollo y la educación de los hijos y, por otro, cambiar diversos tipos de creencias tales como los valores, las actitudes educativas, las teorías implícitas acerca del desarrollo y la educación y/o la percepción del comportamiento de los padres y de los hijos en sus relaciones.

\section{D) Resolver problemas}

El cuarto tipo de objetivos que frecuentemente se persigue en los grupos de formación de padres es la aplicación de la resolución de problemas. Requiere que el orientador proporcione una continua estimulación y retroalimentación constructiva durante el proceso a los padres. El objetivo es enseñar a los padres el paradigma básico de la resolución de problemas que es aplicable a una variedad de problemas del desarrollo de los niños. Las fases componentes del proceso de resolución de problemas incluye las siguientes: a) Identificar el problema, b) Determinar de quién es el problema, 
c) Explorar opciones alternativas de actuación, d) Anticipar los resultados probables de esas acciones, e) Seleccionar y poner en marcha un plan y f) Evaluar la efectividad del plan puesto en marcha.

Cuadro $n^{\circ}$ 2: Propuesta de Fine sobre los objetivos de los programas de formación de padres (Fine, 1991 cit. Fine y Henry, 1991:7)

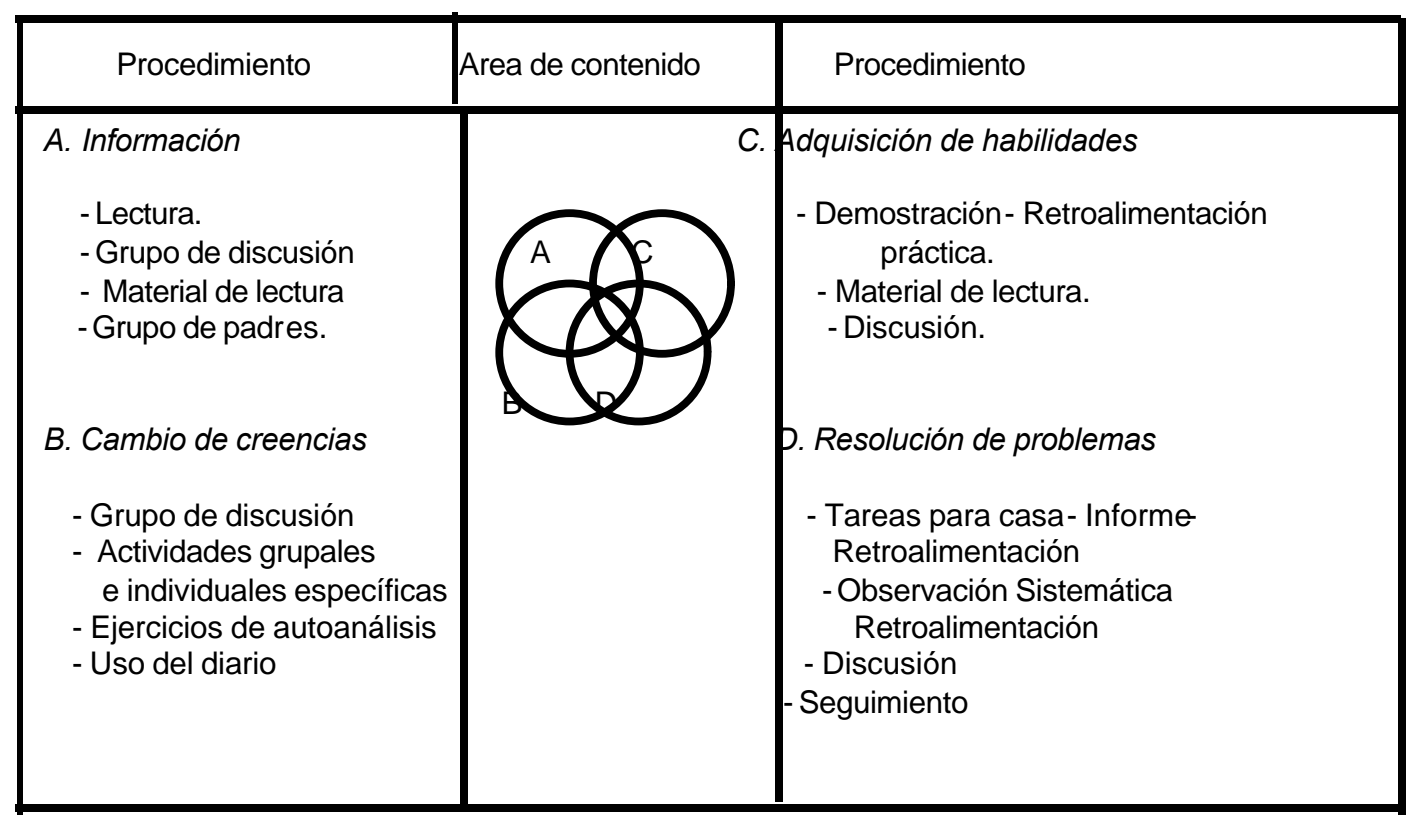

Como se puede apreciar en el cuadro $\mathrm{n}^{\circ}$ 2, estas áreas de objetivos son entidades superpuestas y cualquier orientador deberá decidir el peso relativo de cada una de ellas teniendo en cuenta las necesidades y composición del grupo, la naturaleza de las valores que van a ser transmitidos y que unos tipos de programas se centran más en un tipo de objetivos que en otros (Fine y Henry,1991). Por ejemplo, los programas para padres de bebés con frecuencia se centran exclusivamente en compartir información sobre el desarrollo, la estimulación y la salud; los conductuales y adlerianos destacan el proporcionar información y desarrollar habilidades; por su parte, los programas derivados de la orientación psicoanalítica persiguen en mayor medida incrementar la auto-conciencia sobre el funcionamiento intrapsíquico de los padres (Reisinger, Ora y Frangia, 1976).

\section{Rol del orientador}

El orientador es uno de los componentes críticos de la eficacia de los programas de formación de padres (Arcus y Thomas, 1993; Ben-Peretz, 1990; Cataldo, 1991; Gamson, Hornstein y Borden, 1991). Adoptando el modelo social de la formación de padres (Velazquez y Loscertales, 1987), en el cuadro $\mathrm{n}^{\circ} 3$ y $\mathrm{n}^{\circ} \mathbf{4}$ se presentan las cualidades, las aptitudes y las funciones a lo largo del desarrollo del programa de los orientadores (Cataldo,1991:119). 
Cuadro $n^{\circ}$ 3: Cualidades y aptitudes de los orientadores en los programas de formación de padres (Cataldo,1991:119).

\begin{tabular}{|c|c|}
\hline CUALIDADES & APTITUDES \\
\hline 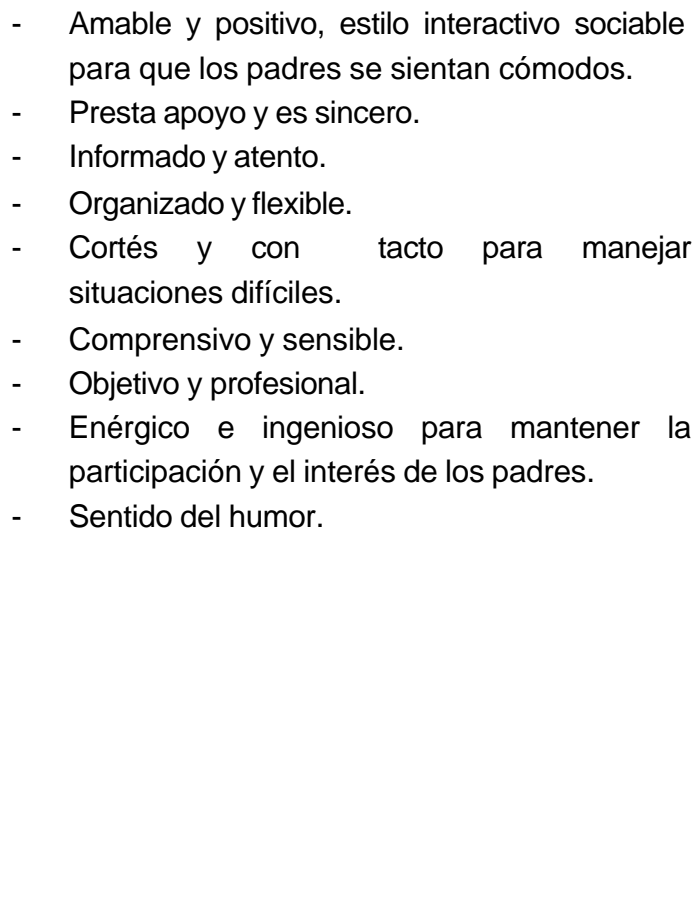 & $\begin{array}{l}\text { - Expectativas realistas. } \\
\text { - Capacidad para la planificación. } \\
\text { - Capacidad de liderazgo democrático. } \\
\text { - Demostración de respeto y aceptación de } \\
\text { los padres y de sus necesidades). } \\
\text { - Capacidad para estimular el diálogo y } \\
\text { fomentar la participación activa. } \\
\text { - Apoyo para ayudar a los padres ante las } \\
\text { críticas, sentimientos contradictorios o } \\
\text { cambio de costumbres y estilos de } \\
\text { patemidad. } \\
\text { - Modelado positivo. } \\
\text { - Coherencia. } \\
\text { - Reconoce el propio impacto en los } \\
\text { miembros de las familias y busca resolver, } \\
\text { de forma confidencial, cualquier dificultad } \\
\text { interpersonal. } \\
\text { Capacidad de coordinación. } \\
\text { Conocimientos y formación de los } \\
\text { orientadores. }\end{array}$ \\
\hline
\end{tabular}

Cuadro $n^{\circ}$ 4: Funciones de los orientadores en los programas de formación de padres (Cataldo,1991:119)

FUNCIONES

- Comprender las diferentes perspectivas y las necesidades de los padres; su propia historia y cultura; sus puntos de vista cotidianos sobre el desarrollo y la educación de los hijos; sus preferencias, objetivos y valores.

- Estimular la reflexión compartida en el grupo sin dar consejos o recetas.

- Delimitar desde el comienzo y a lo largo del programa las expectativas sobre los resultados del programa.

- Delimitar los roles que van a asumir los padres en el programa; receptores de información; participantes activos de las estrategias y métodos de aprendizaje; asesores y supervisores del proyecto en sus hogares; paraprofesionales; miembros del grupo.

- Facilitar el respeto mutuo entre los integrantes del grupo.

- Destacar los puntos fuertes, aspectos positivos que aporta cada participante en un proceso continuo de retroalimentación positiva y constructiva.

- Admitir las limitaciones propias del orientador por falta de pericia en determinadas cuestiones.

- Establecer las normas básicas del funcionamiento en el programa; actitud abierta de respeto hacia los otros, mantenimiento de la intimidad en los grupos ya que se comparte información privada.

- Estimular la participación de todos los participantes. 


\section{Captación de los padres}

En el cuadro $n^{0} 5$ se recogen algunos de los procedimientos utilizados para contactar con los padres y obtener su participación en los programas de formación (Cataldo, 1991:122-125).

Cuadro $n^{\circ}$ 5: Estrategias de captación de los padres en los programas de formación (Cataldo,1991)

\section{ESTRATEGIAS DE CAPTACIÓN DE LOS PADRES}

- Circulares.

- Tableros de anuncios.

- Contacto personal.

- Conferencias.

- Entrevistas.

- $\quad$ Utilización de medios de comunicación (radio, televisión, periódicos, revistas).

- Redes sociales de padres.

- Promoción a través de la ayuda de profesionales sanitarios, asistentes sociales, educadores y de la salud mental.

- Presentación del programa.

- $\quad$ Grupos de asesoramiento a los padres.

- Actos infantiles: Representaciones teatrales, exposiciones fotográficas, excursiones, competiciones deportivas, exposición de manualidades y artes plásticas.

- Sala de reuniones para padres.

- Programas de protección sanitaria.

- Visitas hogar-escuela.

\section{Dificultades}

En el cuadro nํ 6 se presentan las principales dificultades en el desarrollo de los programas de formación de padres (Cataldo, 1991:142-145).

Cuadro n 6: Dificultades de los programas de formación de padres (Cataldo, 1991:142-145).

\section{DIFICULTADES DE LOS PROGRAMAS DE FORMACIÓN DE PADRES}

a) Captación inicial

- Problemas de tiempo, horarios y obligaciones laborales y familiares de los participantes.

- Escasa comprensión de los objetivos por parte de los padres.

- Experiencias anteriores negativas.

- Escasa adecuación a las necesidades delos padres.

- Reticencias y temores ante la institución o centro.

- Temor ante al cambio. 
- Programaciones confusas.

b) Conflictos de puntos de vista y valores

- Conflicto con los valores que subyacen al programa.

- Conflicto entre los participantes y/o el orientador.

c) Personal con formación y aptitudes inadecuadas

- $\quad$ Falta de experiencia con grupos de padres, con adultos o con determinados tipos de familias.

- Escasas habilidades de comunicación.

- Falta de habilidades en resolución de conflictos.

d) Disminución del interés por parte de los padres

- $\quad$ Disminución del número de participantes con el tiempo.

- $\quad$ Expectativas no realistas o inadecuación de los contenidos.

- Escasos recursos materiales o de personal.

e) Problemas en el funcionamiento de los grupos:

- Escasa confidencialidad por parte de profesionales o participantes.

- Dinámicas interpersonales inadecuadas.

Monopolización de la conversación.

- Desafíos entre los miembros del grupo o al guía en torno a creencias o actuaciones.

- Desviaciones en el hilo de la conversación.

- Formación de coaliciones competitivas entre los padres.

- Negarse a participar.

f) Problemas en la escuela o entidad

- Escasos o inadecuados recursos de personal o materiales.

- Conflictos con el personal de la escuela o entidad.

g) Falta de continuidad

- Escasa duración del programa.

- $\quad$ Falta de regularidad en la asistencia por parte de los participantes.

\section{Evaluación de la eficacia de los programas}

La mayoría de los autores de los programas de formación de padres que han presentado sus datos sobre la evaluación de los programas, destacan efectos positivos de los mismos (Medway, 1991). La investigación aporta resultados de la eficacia de los programas de formación de padres en los siguientes aspectos (Cataldo 1991:36):

1) Mejora del desarrollo de los niños

2) Mejora de las relaciones familiares

3) Mayor sentimiento de satisfacción y autocompetencia de los padres en torno a sus tareas y responsabilidades como padres y madres. 
Bartau, Isabel; Maganto, Juana; Etxeberría, Juan: Los Programas de Formación de Padres....

4) Mejora en las relaciones familia-escuela-comunidad.

No obstante, se han destacado diversas dificultades metodológicas de la evaluación de estos programas como procedimientos de muestreo poco rigurosos, medidas muy poco sistemáticas, ausencia de grupos control y escasez de estudios de seguimiento de los efectos a largo plazo (Brock y otros, 1993; Dembo, Sweitzar y Lauritzen, 1985; Boutin y Durning, 1997).

\section{Tipos de Programas de Formación de Padres}

Actualmente existe una amplia literatura sobre formación de padres (estudios de investigación, descripciones de programas, informes de evaluación) como lo demuestra la cantidad de trabajos que se recogen, por ejemplo, en el Centro de Información de Recursos Educativos (ERIC) que se aproxima a las 3000 citas (Brock, Oertwein y Coufal, 1993). Las clasificaciones de los tipos de programas de formación de padres son diversas. Cataldo (1991:96), atendiendo a las áreas de contenido de los programas para la paternidad, diferencia los siguientes:

1) Propuestas informativas (Información sobre el estudio del niño; Salud y nutrición; Información teórica; Situaciones familiares especiales).

2) Propuestas conductuales (Resolución de problemas, Control del comportamiento; Consecuencias lógicas; Establecimiento de límites; Asertividad.

3) Propuestas centradas en la personalidad y la salud mental (Apoyo personal; Comunicación; Valores/desarrollo moral; Aceptación y estima; Aceptación mutua).

4) Propuestas evolutivas (Participación en el juego; Guía sobre el desarrollo; Currículo para el desarrollo; Desarrollo padres -familia).

Otros autores se han basado en el tipo de formatos de instrucción (Brock y otros, 1993; Harmin y Brim, 1980) diferenciando entre: 1) Individual asociado en el modelo de counseling, 2) Grupal y $3^{\circ}$ ) Difusión a través de medios de comunicación (publicaciones divulgativas, radio y televisión).

Otros los han clasificado en función del tipo de padres y necesidades/problemas que se intentan cubrir (Boutin y Durning, 1997: 87-198) diferenciando los siguientes tipos:

1) Programas destinados a los padres de hijos con deficiencias psíquicas, físicas o sensoriales.

2) Programas destinados a padres con niños/adolescentes difíciles.

3) Programas destinados a padres en dificultades personales o sociales.

4) Programas preventivos que, a su vez, se diferencian en función de los enfoques teóricos que subyacen a los programas, en los siguientes (Boutin y Durning, 1997:167-197):

a) Trabajo sobre los comportamientos, centrados en la modificación de conducta.

b) Trabajo sobre la dimensión interrelacional (Enfoque Humanista, Enfoque

Adleriano, Enfoque ecosistémico).

c) Trabajo sobre los procesos cognitivos. 
También se han clasificado atendiendo al alcance social, grado de institucionalización y participación de las familias y sus hijos en los siguientes tipos (Vila, 1997:505):

1) Programas destinados a la formación general de los padres.

2) Programas instruccionales dirigidos a padres.

3) Programas dirigidos a conseguir una mayor implicación de las familias y los educadores en el proceso educativo de los niños.

4) Servicios dirigidos al desarrollo de capacidades infantiles y de competencias educativas en las familias.

\section{La formación de padres y madres: una experiencia educativa en el País Vasco (España)}

El trabajo que se presenta a continuación es el resultado de diversas investigaciones e intervenciones, desde la perspectiva preventiva y comunitaria, dirigidas a la formación de padres y de madres llevadas a cabo en el País Vasco durante la última década por nuestro equipo de investigación. Se han utilizado los programas de intervención familiar de Dinkmeyer y Mckay $(1980,1981,1988)$ porque consideramos que respondían de forma adecuada a las necesidades de formación demandadas por los padres para el desarrollo de la interacción y comunicación familiar y para la estimulación de la cooperación familia-escuela. A continuación, se presentan las características principales del programa originario Systematic Training for Effective Parenting (STEP) (Padres Eficaces Con Entrenamiento Sistemático, (PECES)) de Dinkmeyer y McKay (1976/1981) así como los trabajos de intervención y evaluación llevados a cabo.

\section{Características del programa}

El objetivo principal del PECES/STEP es enseñar a los padres estrategias de comunicación y de resolución de conflictos para mejorar las relaciones padres-hijos en edad infantil. Se centra en la estimulación y la construcción positiva de las relaciones familiares (Fine, 1980). Siguiendo las ideas de Adler (1930,1963), ofrece una teoría explicativa de las relaciones padres-hijos que se basa en que el comportamiento es propositivo y que los padres necesitan identificar el objetivo del comportamiento adecuado e inadecuado de los hijos para poder comprenderlo y facilitar el comportamiento adecuado y las relaciones familiares positivas. Los cuatro objetivos básicos del comportamiento inadecuado son obtener atención, poder, revancha y demostrar insuficiencia.

Presenta diversos métodos para mejorar las relaciones padres-hijos. Un método de motivación, la estimulación del comportamiento positivo de los hijos para desarrollar la confianza en sí mismo y el auto-control. Un método de comunicación abierta que comprende estrategias como la atención reflexiva, los mensajes-yo y técnicas de resolución de problemas como la exploración de alternativas. Un método de disciplina democrático que, frente al castigo, propone la utilización de las consecuencias naturales y lógicas y estimula la participación de todos los miembros de la familia en la toma de decisiones familiares.

Es un programa estructurado que consta del Libro del Guía, del Libro de Padres y el Material Audiovisual. El Libro del Guía ofrece una descripción detallada de los objetivos, los contenidos, la 
Bartau, Isabel; Maganto, Juana; Etxeberría, Juan: Los Programas de Formación de Padres....

metodología y el papel del monitor durante las sesiones. El Libro de Padres contiene lecturas generales de los contenidos, ejercicios prácticos, resúmenes de los conceptos principales y registros de evaluación. El Material Audiovisual incluye cassettes que contienen ejemplos de los principales conceptos y ejercicios prácticos basados en escenas cotidianas de relaciones padres-hijos y transparencias correspondientes a los resúmenes de los conceptos principales de cada una de las sesiones. Se dirige a grupos de padres, entre 20-25 participantes y consta de 9 sesiones de 2 horas de duración.

Los contenidos del PECES correspondientes a las 9 sesiones del programason los siguientes:

1) Comprensión del comportamiento adecuado e inadecuado de los hijos

2) Mayor comprensión acerca de su hijo y acerca de usted mismo como padre

3) La estimulación: construcción de la confianza en sí mismo y de los sentimientos de autoestima de su hijo

4) Comunicación: cómo escuchar a su hijo

5) Comunicación: cómo explorar alternativas y expresar sus ideas y sentimientos a sus hijos

6) Consecuencias naturales y lógicas: un método de disciplina que desarrolla la responsabilidad

7) Aplicación del método de las consecuenciasnaturales y lógicas

8) La reunión familiar

9) Desarrolle la confianza en Vd. mismo y use su potencial

La metodología del programa es participativa y activa. Ofrece materiales teóricos y ejercicios prácticos para que los padres practiquen las estrategias tanto en las sesiones como en el hogar. Se requiere monitor especializado dinamizador de los grupos de padres. La formación de los monitores es un aspecto crucial para la eficacia de estos programas (Gamson, Hornstein y Borden, 1991; King, 1979; Soltz, 1967)

Con respecto a la evaluación del programa PECES/STEP, como afirman Brock y otros (1993) es uno de los programas que más investigación ha suscitado como se muestra en los 51 estudios disponibles en la American Guidance Service (1991). La mayoría de los estudios incluyen diseños pretestpostest, con grupo experimental y grupo control, utilizan medidas objetivas, se ha examinado su utilización con diferentes grupos de padres (abusivos, con hijos con transtornos de aprendizaje y con hijos con problemas de conducta) y los resultados en general han sido positivos (Brock y otros, 1993:98). Asimismo estos autores informan de que los resultados más significativos se refieren a cambios positivos en la interacción padres-hijos (7 de 8 estudios), las actitudes parentales (10 de 13 estudios), las conductas de los hijos (9 de 10 estudios) y las percepciones de los padres de las conductas de sus hijos (11 de 15 estudios) e indican una mayor mejora del clima familiar cuando ambos padres asisten a las sesiones (American Guidance Service, 1991 cit. Brock y otros, 1993: 98). Otros estudios también apuntan resultados positivos como en las actitudes de los padres y de los hijos en sus relaciones (Christensen y Thomas, 1980). 


\section{Una experiencia educativa}

El programa PECES/STEP ha sido objeto de investigación por parte de nuestro equipo en varios municipios del País Vasco. Comenzamos llevándolo a la práctica para responder a una demanda de formación de padres de un centro de Educación Permanente de Adultos, después de los profesores y de la Asociación de Padres de Alumnos de un Centro educativo Público y, posteriormente del Departamento de Bienestar Social de un municipio, experiencia que comenzó en 1993 y debido a su aceptación se prolongó hasta 1999.

El objetivo era poner en marcha y evaluar las posibilidades del programa en nuestro contexto sociocultural mientras respondíamos a demandas muy concretas de formación de padres para mejorar las relaciones padres-hijos. Hemos trabajado tanto con padres de niños en edad infantil (Bartau e Imbert, 1990) como con grupos de padres de adolescentes utilizando el STEP/TEEN que previamente habíamos adaptado (Bartau y Maganto, 1994).

Después adaptamos el STET (Systematic Training for Effective Tteaching (STET) de Dinkmeyer, McKay y Dinkmeyer,1980) versión del PECES/STEP para profesores y llevamos a cabo una intervención conjunta dirigida tanto a los profesores como a los padres de todos los centros escolares (público, privado e ikastola) del municipio (Maganto, Bartau, de la Caba, 1996, 1997, 1998).

Asimismo se ha utilizado el programa PECES/STEP como una herramienta para fomentar la participación de los padres en los centros escolares, comparando los resultados en dos comunidades autónomas (Bartau, Maganto, Etxeberría y Martínez, 1999; Maganto, Bartau, Echeberría y Martínez, 2000). El trabajar con madres y padres durante estos años ha sido una experiencia continua de aprendizaje muy gratificante, que difícilmente se puede reflejar en estas líneas, pero que nos permite afirmar que la formación de padres y madres es un medio para desarrollar habilidades y, en última instancia, trabajar desde y para la prevención (Maganto y Bartau,1999).

La intervención y la evaluación de estos programas nos permiten concluir (Bartau, Maganto, Etxeberría, Martínez, 1999: 50-51) que inciden positivamente en 4 dimensiones principales:

\section{1) Aprendizaje de Procedimientos:}

Los padres son más capaces de identificar cuáles son las estrategias que favorecen/obstaculizan la comunicación en el ambiente familiar. Manifiestan mayor capacidad para seleccionar las estrategias más adecuadas para afrontar los diferentes retos que se les presentan en su vida cotidiana.

A través de la atención reflexiva, aprenden el significado y la utilidad de saber escuchar y comprender el punto de vista de los hijos. Ante el comportamiento inadecuado de los hijos, los padres después del programa cuentan con mayores recursos para expresar los sentimientos negativos al solicitarles un cambio de comportamiento de forma respetuosa o, en su caso, permitir que sus hijos experimenten las consecuencias de sus actos.

También desarrollan una mayor comprensión hacia los hijos y hacia sí mismos como padres, una aceptación más positiva y precisa de sus capacidades y limitaciones y aprenden a cesarrollar como 
padres "el coraje de ser imperfectos". Se aprecia un cambio del nivel de expectativas y exigencias con respecto a sus hijos y, concretamente, de la búsqueda de la perfección y el reconocimiento del resultado pasan a valorar el esfuerzo y la contribución de los hijos cuando hacen las cosas "lo suficientemente bien" para sus posibilidades.

Asimismo, aprenden a transmitir a los hijos confianza en sus posibilidades, a centrarse y reconocer lo positivo, a estimular su responsabilidad y su implicación en la toma de decisiones familiares para favorecer el desarrollo de la confianza en sí mismos. Y, por último, los padres aprenden a discernir entre los problemas que son de su responsabilidad y los problemas que son competencia y responsabilidad de sus hijos y que deben aprender a asumirlos.

\section{2) Control del Comportamiento:}

Los padres aprenden a identificar los objetivos del comportamiento inadecuado de sus hijos y perciben nuevas respuestas de afrontamiento. Por un lado, controlan en mayor medida las reacciones estereotipadas que alientan los conflictos como, por ejemplo, el reforzar las luchas por el poder o el darse por vencidos ante la demostración de insuficiencia de los hijos. Por otro, cuentan con respuestas alternativas para afrontar estas stuaciones y se sienten más capaces de retirarse del conflicto ante las luchas por el poder y de estimular el esfuerzo y el progreso, por pequeño que sea, ante la demostración de sentimientos de insuficiencia de los hijos.

3) Percepción de los padres del Ambiente Familiar:

La percepción de los padres del ambiente familiar se torna más democrática posibilitando la implicación de todos los miembros de la familia en la toma de decisiones. Son más proclives a permitir que los hijos decidan de forma autónoma yasuman progresivamente la responsabilidad y el control sobre su propia vida.

4) Percepción de los padres de la Eficacia del Programa:

Es muy satisfactoria, los padres valoran tanto los aspectos relacionales generados en la dinámica del grupo como los contenidos temáticos abordados en las sesiones de trabajo.

En síntesis, los padres consideran que el programa incide en las siguientes dimensiones (Maganto, Bartau, Etxeberría y Martínez, 2000):

1) Incrementa la motivación por el aprendizaje y la formación de los padres.

2) Estimula la reflexión personal y compartida con el grupo

3) Facilita una mejor comprensión de los hijos adolescentes.

4) Comprende un conjunto de estrategias y procedimientos útiles y eficaces para la mejora de las relaciones padres-hijos.

5) Posibilita con su metodología una adecuada dinámica de participación de los padres en las sesiones de trabajo, aspecto muy valorado por éstos. 
Bartau, Isabel; Maganto, Juana; Etxeberría, Juan: Los Programas de Formación de Padres....

6) Es muy recomendable para otros padres.

Por último, precisar que los beneficios de la utilización de estos programas se incrementan cuando se integran en un programa más amplio basado en las relaciones familia-escuela-comunidad (Maganto, Bartau, de la Caba, 1996,1997,1998). La escuela y las instituciones comunitarias tienen los recursos y las condiciones necesarias para fomentar la intervención educativa a través de los programas de formación de padres y de madres como una estrategia educativa y preventiva por excelencia. Nos corresponde a todos los educadores implicarnos en la cooperación familia-escuelacomunidad para responder al reto educativo y social de la educación de los niñ@s conjunta y coordinadamente.

\section{BIBLIOGRAFÍA}

ADLER, A. (1930): The education of children. New York: Greenberg.

ADLER, A. (1963): The practice and theory of Individual Pschology. Paterson, nj : Littlefield, Adams.

AMERICAN GUIDANCE SERVICE (1991): STEP research studies. Circle Pines, MN: American Guidance Service.

ARCUS, M. E. y THOMAS, J. (1993): The nature and practice of Family Life Education. En M. E. Arcus, J.D. Schvaneveldt y J.J. Moss (Ed.), Handbook of Family Life Education. The practice of Family Life Education. Vol 2 (1-32). Newbury Park: Sage.

BARTAU, I (1992): Intervención familiar: una reflexión acerca de las investigaciones actuales en torno a las relaciones familia-escuela. $V$ Seminario Iberoamericano de Orientación. A.E.O.E.P. (362-367).

BARTAU, I. e IMBERT, C. (1990): Aplicación y evaluación del programa de intervención P.E.C.E.S de Dinkmeyer y McKay en el País Vasco. Revista de Investigación Educativa, Vol.8, № 16 (333-335).

BARTAU, I. y MAGANTO, J.M. (1994): Intervención psicopedagógica familiar: adaptación, aplicación y evaluación del programa S.T.E.P. /adolescencia de Dinkmeyer y Mckay, en el País Vasco. Revista de Investigación Educativa № 23 (344-351).

BARTAU, I., MAGANTO, M, ETXEBERRIA, J., y MARTINEZ, R. (1999): La implicación educativa de los padres: Un programa de formación. Revista Española de Orientación y Psicopedagogía, 10 (17), 1ํㅡ Semestre (43-52).

BEN-PERETZ, M. (1990): The teacher-curriculum encounter: Freeing teachers from the tyranny of facts. Albany, NY: State University or New York Press.

BROCK, G.W., OERTWEIN, M. y COUFAL, J.D. (1993): Parent Education: Theory, research, and practice. En M. E. Arcus, J.D. Schvaneveldt y J.J. Moss (Ed.), Handbook of Family Life Education. The practice of Family Life Education. Vol 2 (87-114). Newbury Park:Sage.

BROMWICH, R. (1981): Working with parents and infants: An interactional approach. Baltimore, MD: University Park Press.

BRUNET, J.J. y NEGRO, J.L. (1985): ¿Cómo organizar una escuela de padres? Tomos I y II. Madrid :S. Pío X.

BOUTIN, G. Y DURNING, P. (1997): Intervenciones socioeducativas en el medio familiar. Madrid: Narcea.

CATALDO, C.Z. (1991): Aprendiendo a ser padres: conceptos y contenidos para el diseño de programas de formación de padres. Madrid : Visor.

CHRISTENSEN, O. y THOMAS, C. (1980): Dreikurs and the search for equality. En M.F. Fine (Ed.), Handbook on parent education. New York: Academic Press.

DEMBO, M.H.,SWEITZAR,M.,LAURITZEN, P. (1985): An evaluation of group parent education: behavioral, PET, and adlerian programs. Review of Educational Research. Vol. 55 No.2 (155-200).

DINKMEYER, D. (1976): The basics of adult-teen relationships. Coral Springs, FL: CMTI Prees.

DINKMEYER, D. y CARLSON, J. (1984a): Time for a better marriage. Circle Pines, MN: American Guidance Service.

DINKMEYER, D. y CARLSON, J. (1984b): Training in Marriage Enrichment (TIME). Circle Pines, MN: American Guidance Service.

DINKMEYER, D. y DINKMEYER, J. (1980): The basics of parenting. Coral Springs, FL: CMTI Prees.

DINKMEYER, D. y LOSONCY, L. (1980): The encouragement book. Englewood Cliffs, NJ: PrenticeHall.

DINKMEYER, D. y McKAY, G. (1973): Raising a responsible child. New York: Simon \& Schuster. 
DINKMEYER, D. y McKAY,G. (1976): Systematic Training for Effective Parenting (STEP): Parent's hanbook. Circle Pines, MN: American Guidance Service (Traducción español (1981): Padres Eficaces con Entrenamiento Sistemático (P.E.C.E.S). Circle Piners, MN: American Guidance Service).

DINKMEYER, D. y McKAY,G. (1983): Systematic Training for Effective Parenting of Teens (STEP/TEEN): Parent's handbook, Leader's guide. Circle Pines, MN: American Guidance Service.

DINKMEYER, D. y McKAY,G. (1989): Parenting young children. Circle Pines, MN: American Guidance Service

DINKMEYER, D., McKAY, G., DINKMEYER, Jr. D.,DINKMEYER, J.y McKAY, J. (1987): The next STEP. Circle Pines, MN: American Guidance Service.

DINKMEYER, D., McKAY, G.y McKAY, J. (1987): New beginnings: Skills for single parents and stepfamily parents. Circle Pines, MN: American Guidance Service

DURNING, P. (1995): Education familiale. Acteurs, processus et enjeux. París: Presses Univeritaires de France.

DURNING, P. (Dir.) (1988): Education familiale. Un panorama des recherches internationales. Paris: Matrice.

FINE, M. J. (Ed.) (1980): Handbook on parent education. New York: Academic Press.

FINE, M. J. (Ed.) (1991): The second handbook on parent education. San Diego: Academic Press.

FINE, M.J. y BROWNSTEIN, C. (1983): Parent education: Problems, promises and implications for school social workers. Social Work in Education, 6 (44-55)

FINE, M.J. y HENRY, S.A. (1991): Professional issues in parent education. En M.J. Fine (Ed.), The second handbook on parent education(3-20). San Diego: Academic Press.

GAMSON, B., HORNSTEIN, H. y BORDEN, B. (1991): Adler-Dreikurs parent study group leadership training. En M.J. Fine (Ed.), The second handbook on parent education (279-304). San Diego: Academic Press.

HARMIN, D. y BRIM, O.G., Jr. (1980): Learning to be parents: Principles, programs and methods. Beverly Hills, CA: Sage.

HICKS, M. y WILLIAMS,J.W. (1981): Current challenges in educating for parenthood. Family Relations: Journal of Applied Family And Child Studies, 30 (579-590).

KING, L. (1979): Stugy group leader's manual to accompany "Teenagers: The continuing challenge". Maple Ridge, British Columbia, Canada: Maple Ridge Family Education Centre.

MAGANTO, J.M. y BARTAU, I. (1999): Intervención familiar ¿es posible la prevención?. Revista Comunidad Educativa, 260 (8-12).

MAGANTO, J.M., BARTAU, I. DE LA CABA, A. (1996): Intervención comunitaria: Un programa de habilidades sociopersonales dirigido a la comunidad educativa. XI Congreso Nacional de Pedagogía, Tomo II (170). San Sebastian.

MAGANTO, J.M., BARTAU, I. DE LA CABA, A. (1997): Intervención para el desarrollo de habilidades sociopersonales en un grupo de profesores. En J.A. Beltrán, V. Santiuste, P. Fernández, A. Tocino y P. Fernández, Nuevas perspectivas en la intervención psicopedagógica: Orientación, educación especial y formación del profesorado. Vol. II, (842-847). Servicio de Publicaciones de la Universidad Complutense de Madrid.

MAGANTO, J.M., BARTAU, I. DE LA CABA, M.A. (1998)iError! Marcador no definido.: Esku-hartze eta ebaluazioa guraso eta irakasleen komunikazio-gaitasunen garapenerako, eta beren eragina semealaba/ikasleen garapen pertsonalean. Revista Tantak, 19 (117-140) Euskal Herriko Unibertsitateko Hezkuntza Aldiskaria, Bilbo.

MAGANTO, J.M., BARTAU, I., ECHEBERRIA, J. Y MARTINEZ, R. (2000): Evaluación de un programa de intervención dirigido a fomentar la participación de la comunidad educativa en los centros escolares. Bordon, 52 (2) (197-211).

MAGANTO,J.M. y BARTAU, I. (1996): Orientación Familiar. Revista de la Asociación Catalana de la Orientación Escolar y Profesional (A.C.O.E.P.), Vol. 8, №15 (19-27).

MAGANTO,J.M. y BARTAU, I.(1994): Intervención familiar: percepción de las prácticas educativas y estrategias de comunicación padres-hijos. En G. Musitu Intervención Comunitaria (177-182). Universidad de Valencia. Cristobal Serrano Villalba, Valencia.

MEDWAY, F. J. (1991): Measuring the effectiveness of parent education. . En M.J. Fine (Ed.), The second handbook on parent education(237-256). San Diego: Academic Press.

OTERO, O.F. (1990): Orientación familiar y orientadores familiares. En V. García Hoz (Dir.), La educación personalizada en la familia (Tratado de Educación Personalizada $n^{\circ} 7$ ) (349-373). Madrid: Rialp.

POURTOIS, J.P. (1984): Eduquer les parents ou comment stimuler la compétence en education. Bruselas: Labor.

REISINGER, J.J. ORA, J.P. y FRANGIA, G.W. (1976): Parents as change agents for their children: A review. Journal of Community Psiychology, 1 (103-123).

SOLTZ,V. (1967): Study group leader's manual for "Children: The Challenge". Chicago: Aldred Adler Institute. 
TAVOILLOT, H. (1982): Educación familiar y educación escolar. En G. Avancini, La pedagogía en el siglo XX (263-273). 3를 ed. Narcea, Madrid.

VELAZQUEZ, M. LOS CERTALES, F.(1987): La escuela de padres. Manual práctico. Alfar, Sevilla.

VILA, I. (1997a): Entorn social i familiar i intervenció psicopedagógica. Barcelona: Universitat Oberta de Catalunya.

VILA, I. (1998): Intervención psicopedagógica en el contexto familiar. En M. J. Rodrigo y J. Palacios (Coords.), Familia y desarrollo humano (501-519). Madrid: Alianza Editorial.

WAPNER (1993): Parental development: A holistic, developmental systems-oriented perspective. En J.Demick, K. Bursik y R. Dibiase, Parental Development. Lawrence Erlbaum Associates, Hillsdale. 